Quasi-Concave Programming

Author(s): Kenneth J. Arrow and Alain C. Enthoven

Source: Econometrica, Vol. 29, No. 4 (Oct., 1961), pp. 779-800

Published by: The Econometric Society

Stable URL: http://www.jstor.org/stable/1911819

Accessed: 02/12/2008 07:48

Your use of the JSTOR archive indicates your acceptance of JSTOR's Terms and Conditions of Use, available at http://www.jstor.org/page/info/about/policies/terms.jsp. JSTOR's Terms and Conditions of Use provides, in part, that unless you have obtained prior permission, you may not download an entire issue of a journal or multiple copies of articles, and you may use content in the JSTOR archive only for your personal, non-commercial use.

Please contact the publisher regarding any further use of this work. Publisher contact information may be obtained at http://www.jstor.org/action/showPublisher?publisherCode=econosoc.

Each copy of any part of a JSTOR transmission must contain the same copyright notice that appears on the screen or printed page of such transmission.

JSTOR is a not-for-profit organization founded in 1995 to build trusted digital archives for scholarship. We work with the scholarly community to preserve their work and the materials they rely upon, and to build a common research platform that promotes the discovery and use of these resources. For more information about JSTOR, please contact support@jstor.org. 


\title{
QUASI-CONCAVE PROGRAMMING
}

\author{
By Kenneth J. Arrow and Alain C. Enthoven
}

We extend present theorems on conditions for a constrained maximum to the case where the maximand and the constraint functions are quasi-concave (e.g., utility functions). Economic applications are briefly discussed.

\section{INTRODUCTION}

OUR PROBLEM is to maximize a differentiable function, $f(x)$, of an $n$-dimensional vector $x=\left(x_{1}, \ldots, x_{n}\right)$, subject to the constraints $g(x) \geqslant 0$, where $g(x)$ is a differentiable $m$-dimensional vector function, $g^{1}(x), \ldots, g^{m}(x)$, and $x \geqslant 0$. H. W. Kuhn and A. W. Tucker, in their frequently quoted paper on Nonlinear Programming [4], proved that if $g(x)$ satisfies their Constraint Qualification, 1 the necessary conditions for $x^{o}$ to maximize $f(x)$ subject to $g(x) \geqslant 0$ and $x \geqslant 0$ (the Kuhn-Tucker-Lagrange conditions, or KTL) are

(KTL)

$$
\begin{aligned}
f_{x}^{o}+\lambda^{o} g_{x}^{o} & \leqslant 0, \\
x^{o}\left(f_{x}^{o}+\lambda^{o} g_{x}^{o}\right) & =0, \\
\lambda^{o} g\left(x^{o}\right) & =0, \\
\lambda^{o} & \geqslant 0,
\end{aligned}
$$

where, for example, $f_{x}^{o}$ is the vector of partial derivatives of $f(x)$ evaluated at the point $x^{0 .}{ }^{2} \mathrm{Kuhn}$ and Tucker also proved that if $f(x)$ and $g(x)$ are concave functions, (KTL) are sufficient conditions for a constrained maximum.

A function is concave if the chord joining any two points on any plane profile of its graph lies everywhere on or below the function. That is, $f(x)$ is concave if

$$
f\left[\theta x+(1-\theta) x^{o}\right] \geqslant \theta f(x)+(1-\theta) f\left(x^{o}\right) \quad(0 \leqslant \theta \leqslant 1)
$$

for all points $x$ and $x^{\circ}$ in the region of definition of $f(x)$. Write (1.1) in the form

$$
\frac{f\left[x^{o}+\theta\left(x-x^{o}\right)\right]-f\left(x^{o}\right)}{\theta} \geqslant f(x)-f\left(x^{o}\right) \quad(0<\theta \leqslant 1)
$$

1 See [4, pp. 483-484]; also [1].

2 In general, we denote by subscripts partial differentiation with respect to the indicated arguments, and a superscript $o$ means evaluation at the point $x^{o}$. Then $g_{x}^{o}$ is the $m \times n$ matrix of partial derivatives of the functions $g^{j}(x)$ with respect to the variables, $x_{1}, \ldots, x_{n}$, evaluated at $x=x^{\circ} ; \lambda^{\circ}$ is an $m$-vector of Lagrange multipliers, and $\lambda^{\circ} g_{x}^{o}$ is an $n$-vector. 
and take the limit of the left-hand side as $\theta \rightarrow 0,{ }^{3}$ to obtain

$$
f_{x}^{o}\left(x-x^{o}\right)+f\left(x^{o}\right) \geqslant f(x)
$$

which is an alternative definition of concavity for differentiable functions. The inequality (1.3) states that if $f(x)$ is concave, it lies everywhere on or below its tangent planes.

A function is quasi-concave if, for each real number $c$, the set $x$ defined by the inequality

$$
f(x) \geqslant c
$$

is convex. That is, $f(x)$ is quasi-concave if

$$
f(x) \geqslant f\left(x^{o}\right) \quad \text { implies } \quad f\left[\theta x+(1-\theta) x^{0}\right] \geqslant f\left(x^{o}\right)
$$

for $0 \leqslant \theta \leqslant 1$. Now, for any $x$ satisfying (1.5), let

$$
F(\theta)=f\left[\theta x+(1-\theta) x^{o}\right] \geqslant f\left(x^{o}\right)=F(0) .
$$

Therefore, $F^{\prime}(0) \geqslant 0$. Thus, differentiating $F(\theta)$ and setting $\theta$ equal to zero, we have

$$
f(x) \geqslant f\left(x^{0}\right) \quad \text { implies } \quad f_{x}^{o}\left(x-x^{o}\right) \geqslant 0
$$

for differentiable quasi-concave functions. 4

In speaking of a quasi-concave function, some specific domain of definition, taken to be a convex set, is assumed. Thus, the function $x_{1} x_{2}$ is quasi-concave for nonnegative $x_{1}, x_{2}$, but not for all $x$. A function which is quasi-concave for a convex domain of definition cannot necessarily be extended to a quasiconcave function over the entire space. (In the same circumstances, a concave

3 See [4, pp. 485-486]. A function, $f(x)$, of several variables is differentiable if $f(x+h)=f(x)+c h+e h$, where $c$ is a vector depending on $x$ but not on $h$ and $e$ is a vector which goes to zero with $h$. If a function is differentiable, then its partial derivatives exist, and $f_{x}=c$, but the existence of the partial derivatives does not necessarily imply differentiability; see [2, pp. 59-61]. In particular, $f\left[x^{o}+\theta\left(x-x^{o}\right)\right]=f\left(x^{o}\right)+$ $f_{x}^{o} \theta\left(x-x^{o}\right)+\varepsilon \theta$, where $\varepsilon=e\left(x-x^{o}\right)$ goes to zero with $\theta$. Then,

$$
\frac{f\left[x^{0}+\theta\left(x-x^{o}\right)\right]-f\left(x^{o}\right)}{\theta}=f_{x}^{o}\left(x-x^{0}\right)+\varepsilon \rightarrow f_{x}^{o}\left(x-x^{o}\right) \text { as } \theta \rightarrow 0 .
$$

Here $f_{x}^{o}\left(x-x^{o}\right)$ is an inner product of the two vectors.

4 The differentiation with respect to $\theta$ is, in effect, taking the directional derivative of $f(x)$ at $x^{\circ}$ in the direction of the point $x$. It is clear from the definition of quasiconcavity that this derivative, $f_{x}^{o} \frac{x-x^{o}}{d}$, where the terms $\frac{x-x^{o}}{d}$ are the direction cosines $\left(d=\left[\Sigma\left(x-x^{o}\right)^{2}\right]^{i}\right)$, must be nonnegative. For a definition of directional derivatives, see [2, pp. 262-263]. Wold [7] defines a function to be convex towards the origin if (1.7) holds (his terminology is geometrically valid in the case he considers, where $\left.f_{x}>0\right)$; since (1.7) can be shown to imply quasi-concavity, the two definitions are equivalent. 
function can always be so extended.) In this paper, we shall usually deal with functions quasi-concave over nonnegative values of the variables.

It is clear from (1.1) that all concave functions are quasi-concave. It also can be shown that any monotonic nondecreasing function of a quasi-concave function-and therefore of a concave function-is quasi-concave. ${ }^{5}$ However, not every quasi-concave function can be expressed as a monotonic nondecreasing function of a concave function. ${ }^{6}$ Thus quasi-concavity is a generalization of the notion of concavity.

In terms of traditional economic theory, a concave function is one that satisfies the second order conditions for a maximum, that is,

$$
d^{2} f=\sum_{i=1}^{n} \sum_{j=1}^{n} f_{x_{i}} x_{j} d x_{i} d x_{j} \leqslant 0 .
$$

Quasi-concavity is a weaker condition; (1.8) does not have to hold for quasiconcave functions. ${ }^{7}$ A quasi-concave function is one that has a diminishing marginal rate of substitution if $f_{x}>0$, or an increasing marginal rate of transformation if $f_{x}<0$, between any pair of variables, or between any distinct composite variables. Let $x^{0}$ and $x^{1}$ be any two nonnegative vectors not zero and not proportional to each other. Then, if we let

$$
g(u, v)=f\left(u x^{0}+v x^{1}\right), \quad u \geqslant 0, v \geqslant 0,
$$

if $f(x)$ is quasi-concave and twice differentiable. It can also be shown that if (1.10) holds everywhere, $f(x)$ is quasi-concave.

Alternatively, if $f(x)$ is quasi-concave, $(-1)^{r} D_{r} \geqslant 0$, for $r=1, \ldots, n$ and for all $x$, where $D_{r}$ is the bordered determinant

$$
D_{r}=\left|\begin{array}{cccc}
0 & f_{x_{1}} & & f_{x_{r}} \\
f_{x_{1}} & f_{x_{1} x_{1}} & \ldots & f_{x_{1} x_{r}} \\
\vdots & \vdots & & \vdots \\
f_{x_{r}} & f_{x_{r} x_{1}} & \ldots & f_{x_{r} x_{r}}
\end{array}\right|
$$

5 (1.5) can be written $f\left[\theta x+(1-\theta) x^{0}\right] \geqslant \min \left[f(x), f\left(x^{0}\right)\right]$. Let $\phi$ be a monotonic nondecreasing transformation. Then $\phi$ does not reverse rankings. That is, $f(x) \geqslant f\left(x^{\circ}\right)$ implies $\phi[f(x)] \geqslant \phi\left[f\left(x^{\circ}\right)\right]$. Therefore, $\phi\left\{f\left[\theta x+(1-\theta) x^{0}\right]\right\} \geqslant \phi\left\{\min \left[f(x), f\left(x^{0}\right)\right]\right\}$. Since $\phi$ does not reverse rankings, $\phi\left\{\min \left[f(x),\left[f\left(x^{\circ}\right)\right]\right\}=\min \left\{\phi[f(x)], \phi\left[f\left(x^{o}\right)\right]\right\}\right.$ whence $\phi[f(x)]$ is quasi-concave.

${ }_{6}^{6}$ For example, $f(x, y)=(x-1)+\left[(1-x)^{2}+4(x+y)\right]^{\frac{1}{2}}$ is quasi-concave. Its contour lines are straight lines that are not parallel. See also the example at the end of Section 2, below. Fenchel [3, p. 134] has proved that such a function cannot be transformed into a concave function by a monotonic nondecreasing transformation.

7 For example, $x_{1} x_{2}$ does not satisfy (1.8). 
Moreover, a sufficient condition for $f(x)$ to be quasi-concave for $x \geqslant 0$ is that $D_{r}$ have the sign $(-1)^{r}$ for all $x$ and all $r=1, \ldots, n .^{8}$

We seek sufficient conditions for $x^{0} \geqslant 0$ to maximize $f(x)$ subject to the constraints $g(x) \geqslant 0$ when $f(x)$ and $g(x)$ are differentiable quasi-concave functions. It is not true that (KTL) alone are sufficient conditions for a constrained maximum, as the following examples illustrate.

Any monotonic function of one variable is clearly quasi-concave. Let

$$
f(x)=(x-1)^{3}, \quad x \geqslant 0,
$$

and maximize it subject to the constraint

$$
g(x)=2-x \geqslant 0 .
$$

If $x^{0}=1, \lambda^{0}=0,(\mathrm{KTL})$ is satisfied, yet clearly the constrained maximum occurs at $x=2$, not $x=1$.

More generally, let $\mathscr{F}(x)$ be any quasi-concave function and $x^{\circ}$ any point, and let

$$
f(x)=\left[\mathscr{F}(x)-\mathscr{F}\left(x^{0}\right)\right]^{3} .
$$

Then $f(x)$ is quasi-concave and has the same maxima as $\mathscr{F}(x)$. But $f_{x}^{o}=0$, although $x^{0}$ was chosen arbitrarily. Moreover, if $g(x)$ is any vector function for which $g\left(x^{0}\right) \geqslant 0$, (KTL) is satisfied if $x=x^{0}$ and $\lambda^{0}=0$, although $x^{o}$ certainly need not be the constrained maximum for $f(x)$ subject to $g(x) \geqslant 0$.

We also state conditions under which (KTL) will be necessary for a constrained maximum, when the constraints are quasi-concave. The following example makes clear the fact that (KTL) are not always necessary conditions, and that some additional condition must be satisfied. Maximize $x_{1} x_{2}$ subject to the constraints $x_{1} \geqslant 0, x_{2} \geqslant 0$ and

$$
g(x)=\left(1-x_{1}-x_{2}\right)^{3} \geqslant 0 .
$$

The constrained maximum occurs at $x_{1}^{0}=x_{2}^{0}=1 / 2$, but there is no value of $\lambda$ for which (KTL) can be satisfied at that point. This example also illustrates the fact that it is the constraint functions and not the constraint set which must satisfy the additional condition, for (1.15) and

$$
1-x_{1}-x_{2} \geqslant 0
$$

define the same convex set. Yet (KTL) are satisfied at $x^{\circ}$ with $\lambda^{\circ}=1 / 2$ when the constraint is (1.16), and, in fact, in this case (KTL) is a necessary condition for a maximum. The Kuhn-Tucker Constraint Qualification is designed to meet the problem. Since it is rather complicated to apply, in Section 3 below, we present a simpler condition on quasi-concave constraints which,

8 These propositions are frequently used in the literature on utility functions, but rigorous proofs starting from the concept of quasi-concavity seem to be lacking. We give such proofs in Section 4, below. 
when satisfied, implies that the Constraint Qualification must be satisfied, and therefore that (KTL) are necessary for a constrained maximum.

\section{SUFFICIENT CONDITIONS FOR A CONSTRAINED MAXIMUM}

Let a relevant variable be one which can take on a positive value without necessarily violating the constraints. Or, more formally, $x_{i_{o}}$ is a relevant variable if there is some point in the constraint set, say $x^{*}$, at which $x_{i_{o}}^{*}>0$. Then we shall prove the following theorem:

THEOREM 1: Let $f(x)$ be a differentiable quasi-concave function of the $n$-dimensional vector $x$, and let $g(x)$ be an m-dimensional differentiable quasiconcave vector function, both defined for $x \geqslant 0$. Let $x^{\circ}$ and $\lambda^{\circ}$ satisfy $(K T L)$, and let one of the following conditions be satisfied:

(a) $f_{x_{i_{o}}}^{o}<0$ for at least one variable $x_{i_{o}}$;

(b) $f_{x_{i_{1}}}^{o}>0$ for some relevant variable $x_{i_{1}}$;

(c) $f_{x}^{o} \neq 0$ and $f(x)$ is twice differentiable in the neighborhood of $x^{o} ; 9$

(d) $f(x)$ is concave.

Then $x^{\circ}$ maximizes $f(x)$ subject to the constraints $g(x) \geqslant 0, x \geqslant 0$.

Only one of these four conditions-and there may be others-need be satisfied for $x^{o}$ to maximize $f(x)$ subject to the constraints, if (KTL) is satisfied at $x^{0 .}{ }^{10}$ Condition (b) will be satisfied if $x_{i_{1}}^{o} f_{x_{i_{1}}}^{o}>0$, if any $f_{x_{i_{o}}}^{o}>0$ and all $x_{i_{o}}$ are relevant (the usual case in economic theory), or if $f_{x}^{o}>0$ and any $x_{i_{o}}$ is relevant. If no $x_{i_{o}}$ is relevant, the problem is trivial. From (a) and (b) it follows that $f_{x}^{o} \neq 0$ is sufficient if all $x_{i}$ are relevant.

Perhaps these conditions can be better understood if we consider what conditions $f(x)$ must satisfy if the theorem does not apply. First, from (d), $f(x)$ must be a quasi-concave function that is not concave; from (a), $f_{x}^{0} \geqslant 0$; from $(\mathrm{b}), f_{x_{i}}^{o}=0$ for all relevant variables. Then from (c), either $f_{x}^{o}=0$, or $f_{x_{i}}^{o}=0$ for all relevant variables and $f(x)$ is not twice differentiable. Thus, (KTL) fails to be sufficient in the case of the cubic transforms shown in Section 1 because $f_{x}^{o}=0$. An example in which (KTL) fails but $f_{x}^{o} \neq 0$ follows this proof.

9 That is, all of the second order partial derivatives of $f(x)$ exist at $x^{\circ}$. However, they may be equal to zero.

10 In fact, we developed a series of conditions on $g(x)$ analogous to conditions (a) through (d), only to discover that the cases in which they added anything to conditions (a) through (d) were vacuous. 
Proof. We use the following identity

$$
f_{x}^{0}\left(x^{1}-x^{0}\right)=\left(x^{1}-x^{0}\right)\left(f_{x}^{0}+\lambda^{0} g_{x}^{0}\right)-\lambda^{0} g_{x}^{0}\left(x^{1}-x^{0}\right) .
$$

If $x^{0}$ satisfies (KTL) and $x^{1}$ is in the constraint set, the first term on the right-hand side is nonpositive. The second term on the right-hand side is also nonpositive under these conditions. If $\lambda_{j}^{o}=0$, the $j$ th component of the term vanishes. If $\lambda_{j}^{o}>0, g^{j}\left(x^{o}\right)=0$, and the fact that $x^{1}$ is in the constraint set, that is $g^{j}\left(x^{1}\right) \geqslant 0$, implies $g^{j}\left(x^{1}\right) \geqslant g^{j}\left(x^{o}\right)$ or, by (1.7), $g_{x}^{j o}\left(x^{1}-x^{o}\right) \geqslant 0$. Therefore, for $f(x)$ and $g(x)$ quasi-concave,

$$
g\left(x^{1}\right) \geqslant 0, x^{1} \geqslant 0 \quad \text { implies } \quad f_{x}^{o}\left(x^{1}-x^{o}\right) \leqslant 0
$$

if $x^{o}$ satisfies (KTL).

(a) $f_{x_{i}}^{o}<0$ for at least one variable $x_{i_{o}}$.

Let $h$ be the unit vector in the $i_{o}$-th direction. ${ }^{11}$ Let $x^{2}=x^{o}+h$. Then

$$
f_{x}^{o}\left(x^{2}-x^{o}\right)=f_{x}^{o} h=f_{x_{i}}^{o}<0, \quad x^{2} \geqslant 0 .
$$

For any $x^{1}$ in the constraint set, let

$$
x^{1}(\theta)=(1-\theta) x^{1}+\theta x^{2}, \quad x^{o}(\theta)=(1-\theta) x^{o}+\theta x^{2} .
$$

Then

$$
\begin{gathered}
f_{x}^{o}\left[x^{o}(\theta)-x^{o}\right]=\theta f_{x}^{o}\left(x^{2}-x^{o}\right)<0 \quad \text { for } \theta>0, \\
f_{x}^{o}\left[x^{1}(\theta)-x^{o}(\theta)\right]=(1-\theta) f_{x}^{o}\left(x^{1}-x^{o}\right) \leqslant 0 \quad \text { for } \theta \leqslant 1,
\end{gathered}
$$

from (2.2). Adding, we find

$$
f_{x}^{o}\left[x^{1}(\theta)-x^{o}\right]<0 \quad \text { for } 0<\theta \leqslant 1,
$$

and from (1.7) this is possible only if

$$
f\left[x^{1}(\theta)\right]<f\left(x^{o}\right) .
$$

As $\theta$ approaches zero, $x^{1}(\theta)$ approaches $x^{1}$, and so $f\left(x^{1}\right) \leqslant f\left(x^{0}\right)$.

(b) $f_{x_{i_{1}}}^{0}>0$ for some relevant variable $x_{i_{1}}$.

If we exclude case (a), $x^{0} f_{x}^{0}>0$ and (b) are equivalent. Clearly, $x^{0} f_{x}^{o}>0$ implies that (b) is satisfied. For the converse, note that, by (2.2),

$$
f_{x}^{o} x^{1} \leqslant f_{x}^{o} x^{o},
$$

$11 h$ is the unit vector in, e.g., the direction of $x_{2}$ if $h=(0,1,0, \ldots, 0)$. 
for all $x^{1}$ in the constraint set. Excluding (a),

$$
f_{x}^{o} \geqslant 0 \text {. }
$$

But (b) implies that for some $x^{*}$ in the constraint set and for some $i_{o}, f_{x_{i_{o}}}^{0}>0$ and $x_{i_{o}}^{*}>0$, which, together with (2.9) and the nonnegativity of $x^{*}$, implies that $f_{x}^{o} x^{*}>0$. If we let $x^{1}=x^{*}$ in (2.8), we find that $f_{x}^{o} x^{o}>0$.

If now we let $x^{2}=0$, we see that (2.3) again holds, and the rest of the argument under (a) is valid.

(c) $f_{x}^{0} \neq 0$ and $f(x)$ is twice differentiable in the neighborhood of $x^{\circ}$.

Partition the vector $x^{o}$ into two sub-vectors, $y^{o}$ and $z^{o}$ corresponding to the relevant and irrelevant variables respectively. Then if we exclude the two cases already covered, but assume $f_{x}^{o} \neq 0$, we have

$$
f_{y}^{o}=0, \quad f_{z}^{o} \geqslant 0, \quad f_{z_{o}}^{o}>0 \quad \text { for some } z_{i_{o}} .
$$

By the definition of an irrelevant variable, $z^{0}=0$ and $z^{1}=0$ for all $x^{1}=$ $\left(y^{1}, z^{1}\right)$ in the constraint set. Therefore, to prove the theorem it is sufficient to prove that $f\left(y^{0}, 0\right) \geqslant f\left(y^{1}, 0\right)$ for all $y^{1} \geqslant 0$.

Define the function

$$
\phi(u, v)=f\left[(1-u) y^{o}+u y^{1}, v \bar{z}\right]-f\left(y^{o}, 0\right)
$$

for $0 \leqslant u \leqslant 1$, and $v \geqslant 0$, for any $y^{1} \geqslant 0$ and for any $\bar{z} \geqslant 0$ such that $\bar{z}_{i_{o}}>0$. Because it is essentially $f(x)$ with the range of variation of $x$ restricted to a convex subset of the nonnegative orthant, $\phi(u, v)$ is quasi-concave. Then we have

$$
\begin{gathered}
\phi(0,0)=0, \\
\phi_{u}(0,0)=f_{y}^{o}\left(y^{1}-y^{o}\right)=0,
\end{gathered}
$$

and

$$
\phi_{v}(0,0)=f_{z}^{o} \bar{z}>0 .
$$

We want to prove $\phi(1,0) \leqslant 0$, or to disprove $\phi(1,0)>0$. To do so, first we shall establish the fact that within a sufficiently small neighborhood of zero, $\phi(u, 0)$ is either positive, zero, or negative (but not more than one of the three). Then we shall show that $\phi(u, 0)=0$ and $\phi(u, 0)<0$ in a neighborhood of zero are incompatible with $\phi(1,0)>0$ while $\phi(u, 0)>0$ contradicts the hypotheses of the theorem.

First, if for some $\bar{u}>0, \phi(\bar{u}, 0) \geqslant 0$, then by quasi-concavity, (1.5), and (2.12), $\phi(u, 0) \geqslant 0$ for all $u$ such that $0 \leqslant u \leqslant \bar{u}$. Thus, either $\phi(u, 0) \geqslant 0$ or $\phi(u, 0)<0$ for all $u$ in the interval. If $\phi(u, 0) \geqslant 0$, either there exists some sequence of points $u_{n}$ approaching zero on which $\phi(u, 0)>0$, or there does 
not. If there does not, $\phi(u, 0)=0$ for $u>0$ sufficiently small. If there does, then, by quasi-concavity and (1.5), $\phi(u, 0)>0$ in the intervals between the points in the sequence, and therefore $\phi(u, 0)>0$ for $u>0$ sufficiently small. Therefore, either $\phi(u, 0)>0$, or $\phi(u, 0)=0$, or $\phi(u, 0)<0$ in a neighborhood of $u=0$.

Clearly, if $\phi(1,0)>0$, by $(1.5), \phi(u, 0) \geqslant 0$ for all $u$ in the interval $0 \leqslant u \leqslant 1$, and $\phi(u, 0)$ cannot be negative.

Now, suppose $\phi(u, 0)=0$ in the neighborhood of 0 . If $\phi(1,0)>0$, we must have

$$
\begin{aligned}
& \phi(u, 0)=0, \quad 0 \leqslant u \leqslant u^{*} ; \\
& \phi(u, 0)>0, \quad u^{*}<u \leqslant 1 ;
\end{aligned}
$$

where $u^{*}>0$. Since, by (2.14), $\phi_{v}(0,0)>0$, there is a solution, $u(v)$, to the equation

$$
\phi[u(v), 0]=\phi(0, v),
$$

with $u(v) \geqslant u^{*}$, for $v$ sufficiently small. The solution may not be unique, but this does not matter. In any case,

$$
\lim _{v \rightarrow 0} u(v)=u^{*} .
$$

Let $\theta=1-u^{*} / u(v)$, and form a combination of the points $[u(v), 0]$ and $(0, v)$ with the weights $1-\theta$ and $\theta$ respectively. Then, (2.16) and (1.5) imply

$$
\phi[(1-\theta) u(v), \theta v]=\phi\left(u^{*}, \theta v\right) \geqslant \phi(0, v) .
$$

By Rolle's Theorem (the law of the mean),

$$
\phi_{v}\left(u^{*}, v^{*}\right)=\frac{\phi\left(u^{*}, \theta v\right)-\phi\left(u^{*}, 0\right)}{\theta v}
$$

for some $v^{*}$ in the interval $0 \leqslant v^{*} \leqslant \theta v$. But $\phi\left(u^{*}, 0\right)=0$, by $(2.15)$, so that (2.18) and (2.19) imply

$$
\phi_{v}\left(u^{*}, v^{*}\right) \geqslant \frac{1}{\theta} \frac{\phi(0, v)}{v} .
$$

Now take the limits of both sides as $v$ approaches zero. By (2.17), $\theta$ approaches zero as $v$ does. $\phi(0, v) / v$ approaches $\phi_{v}(0,0)$ which is positive. Therefore, the right-hand side approaches infinity. Since $\phi_{v}$ is differentiable by hypothesis, it is continuous, so that the left-hand side approaches $\phi_{v}\left(u^{*}, 0\right)$ which is finite. Therefore, the hypotheses lead to a contradiction, and $\phi(u, 0)=0$ for $u>0$ and $\phi(1,0)>0$ are incompatible.

Finally, suppose $\phi(u, 0)>0$ for $u>0$ sufficiently small. Define $u(v)$ as in (2.16). Now

$$
\lim _{v \rightarrow 0} u(v)=0
$$


Consider $\phi(u, v)$ on the line connecting the points $(0, v)$ and $[u(v), 0]$. Since $\phi(u, v)$ is quasi-concave, its value along this line must be greater than or equal to its value at the end points. Therefore, the directional derivative of $\phi(u, v)$ at $(0, v)$ in the direction of $[u(v), 0]$ must be nonnegative. That is

$$
u(v) \phi_{u}(0, v)-v \phi_{v}(0, v) \geqslant 0.12
$$

This can be written as

$$
u(v) \frac{\phi_{u}(0, v)}{v} \geqslant \phi_{v}(0, v) .
$$

Taking limits as $v$, and therefore $u(v)$, approach zero, we obtain $\phi_{v}(0,0)>0$ on the right-hand side. On the left-hand side, the limit of $\phi_{u}(0, v) / v$ as $v$ approaches zero is $\phi_{u v}(0,0)$. The existence of this derivative is, of course, one of the hypotheses of the theorem. The limit of the left-hand side is zero, which is a contradiction. Therefore, $\phi(u, 0)>0$ for $u>0$ sufficiently small contradicts the hypotheses of the theorem, and part (c) of the theorem is proved.

(d) $f(x)$ is concave. ${ }^{13}$

(1.3) and (2.2) imply $f\left(x^{0}\right) \geqslant f\left(x^{1}\right)$ for all $x^{1} \geqslant 0, g\left(x^{1}\right) \geqslant 0$. This completes the proof of Theorem 1 .

Now we shall construct implicitly a differentiable (in fact, continuously differentiable) quasi-concave function that satisfies (KTL) at a point $x^{o}$ with $f_{x}^{0} \neq 0$, but which does not have a constrained maximum at that point. The example is designed to show that although the condition of twice differentiability, condition (c) of the theorem, can be weakened, it cannot be dispensed with altogether.

From the proof, it is clear that such an example must be found in a function $f(x, y)$ with $f_{x}(0,0)=0, f(x, 0)$ positive in a right-hand neighborhood, and $f_{y}(0,0)>0$.

The example will be chosen so that $f(0, y)=y, f_{x}(x, 0)=-1 / \log x$ for $x \leqslant 1 / 2$, and $1 / \log 2$ for $x>1 / 2$. Given the definition of $f(x, y)$ on the two axes, we complete the definition by requiring that all the level curves be straight lines, which insures the quasi-concavity of the example. Formally, for any fixed value of $f(x, y)$, say $z$, we define $X(z)$ as the solution of the equation, $f(x, 0)=z$. Then the level curve $f(x, y)=z$ intersects the $x$-axis at $x=X(z)$ and the $y$-axis at $y=z$. If the level curve is to be a straight line, and $(x, y)$ is any point on it, we have

$$
\frac{x}{X(z)}+\frac{y}{z}=1
$$

12 This is an application of $(1.7)$ to $\phi(u, v)$.

13 This is more general than the Kuhn-Tucker Theorem because the components of $g(x)$ are assumed to be quasi-concave rather than concave. See [4]. 
For fixed $x$ and $y$, then, $f(x, y)$ is the unique positive value of $z$ for which (2.24) is satisfied (except that $f(x, y)=0$ for $x=y=0$ ).

Since $f_{x}(0,0)=0, f_{y}(0,0)=1$, (KTL) is satisfied at the origin for the constraint, $-y \geqslant 0$, with $\lambda^{\circ}=1$. But the origin is not a constrained maximum. It remains only to show that $f_{x}$ and $f_{y}$ are continuously differentiable. The construction makes clear, and it can be shown analytically, that no difficulty could arise except possibly at the origin. The functions $f_{x}$ and $f_{y}$ can be evaluated from (2.24) by implicit differentiation, and careful passage to the limit as $x$ and $y$ both approach zero shows that both are continuous, with $f_{x}$ approaching zero and $f_{y}$ approaching 1 .

Remark. ${ }^{14}$ The hypothesis that the constraint function $g(x)$ be quasi-concave was used only to establish that, for all $x^{1}$ in the constraint set,

$$
g_{x}^{j o}\left(x^{1}-x^{o}\right) \geqslant 0,
$$

for all constraints for which $g^{j}\left(x^{0}\right)=0$. But for this purpose it suffices that the constraint set be a convex set. For then

$$
(1-\theta) x^{o}+\theta x^{1}
$$

belongs to the constraint set for $0 \leqslant \theta \leqslant 1$. By definition of the constraint set,

$$
g^{j}\left[(1-\theta) x^{o}+\theta x^{1}\right] \geqslant 0 \quad \text { for } 0 \leqslant \theta \leqslant 1 .
$$

Further, for $\theta=0$, the left-hand side becomes $g^{j}\left(x^{o}\right)=0$, so that the derivative with respect to $\theta$ at $\theta=0$ must be nonnegative. By the chain rule, this statement is equivalent to (2.25).

\section{NECESSARY CONDITIONS FOR A CONSTRAINED MAXIMUM}

Kuhn and Tucker [4] showed that (KTL) are necessary conditions for a constrained maximum provided the constraint functions $g(x)$ satisfy a condition termed by them the Constraint Qualification. To state the condition, we define a contained path in the direction $\mathscr{E}=\left(\mathscr{E}_{1}, \ldots, \mathscr{E}_{n}\right)$ to be a vector function $\psi(\theta)$, defined for the real variable $\theta \geqslant 0$ in an interval beginning at $\theta=0$, whose values are points in the constraint set, and differentiable at $\theta=0$ with $\psi^{\prime}(0)=\mathscr{E}$. The Constraint Qualification then requires that for any $x^{\circ}$ in the constraint set, there is a contained path with $\psi(0)=x^{\circ}$ in any direction $\mathscr{E}$ satisfying the conditions

$$
\begin{gathered}
\text { if } g^{j}\left(x^{o}\right)=0, \quad \text { then } \quad g_{x}^{j o} \mathscr{E} \geqslant 0, \\
\text { if } x_{i}^{o}=0, \quad \text { then } \quad \mathscr{E}_{i} \geqslant 0.1
\end{gathered}
$$

14 We are indebted for this remark to Hirofumi Uzawa.

15 Kuhn and Tucker [4, p. 483], require the path to be differentiable but a careful reading of their proof (p. 484 ) shows that only differentiability at $\theta=0$ is used. 
To grasp the meaning of these conditions, consider any constraint, $g^{j}(x) \geqslant 0$, effective at $x^{o}$. The tangent hyperplane, $g_{x}^{j o}\left(x-x^{o}\right)=0$, then divides the space into two half-spaces (provided $g_{x}^{j o} \neq 0$ ), one of which contains the constraint set. Then the directions satisfying (3.1) must point into or along the boundary of that half-space. A similar remark applies to the effective nonnegativity constraints. Then the Constraint Qualification requires that for every direction from $x^{o}$ which points into or along the boundaries of the appropriate half-spaces for each effective constraint, there is some path that begins at $x^{o}$ in the direction $\mathscr{E}$ all of whose points in some neighborhood of $x^{o}$ are in the constraint set. As Kuhn and Tucker point out, the Constraint Qualification is designed to rule out such singularities as outward pointing cusps at the boundary of the constraint set at which $\lambda$ 's satisfying (KTL) may not exist.

In [1], some simpler conditions which, when satisfied, imply that the Constraint Qualification is satisfied were studied. One such condition is that $g(x)$ be linear. Another is that $g(x)$ be concave and that for some $x^{*} \geqslant 0$, $g\left(x^{*}\right)>0$ (that is, each coordinate is positive). ${ }^{16}$ If the constraints $g(x)$ arise from a problem in activity analysis, then this condition means that it is possible to reduce all initial availabilities of primary commodities to some extent and still produce a positive amount of each intermediate and final good.

Since we are interested here in quasi-concave constraints, we shall state a generalization of the latter condition. ${ }^{17}$

THEOREM 2: Let $g(x)$ be an m-dimensional differentiable quasi-concave vector function. Let $g\left(x^{*}\right)>0$ for some $x^{*} \geqslant 0$, and for each $j$ let either

(a) $g^{j}(x)$ be concave, or

(b) for each $x^{o}$ in the constraint set, $g_{x}^{j o} \neq 0$.

Then $g(x)$ satisfies the Constraint Qualification.

Therefore, if $x^{o}$ maximizes any differentiable function $f(x)$ subject to $g(x) \geqslant 0$, (KTL) must be satisfied.

If the hypotheses of Theorems 1 and 2 both hold, (KTL) are necessary and sufficient for a constrained maximum.

16 This condition was used by M. Slater [6] in the case in which $f(x)$ is also assumed concave.

17 This is a special case of Theorem 3, Corollary 5 in [1]. A proof of Theorem 2 above appeared in the present manuscript as accepted for publication, but a simpler treatment was subsequently developed in [1]. 


\section{EXTENSIONS OF THE THEOREMS}

(1) Dropping the Nonnegativity Constraints.

If $f(x)$ and $g(x)$ are defined for all $x$, and not just for those values in the nonnegative orthant, the conditions (a), (b), and (c) of Section 2 become merely $f_{x}^{0} \neq 0$, for, in effect, all variables become relevant. That is, in the proof of condition (b), we choose $x^{*}$ so that $f_{x}^{o} x^{*}>0$. If $f_{x}^{o} \neq 0$, this can always be done when $x$ is not restricted to be nonnegative. Thus we can say that (KTL) is sufficient for $x^{o}$ to maximize $f(x)$ subject to $g(x) \geqslant 0$, where $f(x)$ and $g(x)$ are differentiable quasi-concave functions provided that either (a) $f_{x}^{o} \neq 0$, or (b) $f(x)$ is concave.

In this case, the first two lines of (KTL) become simply $f_{x}^{0}+\lambda \circ g_{x}^{0}=0$.

Actually, the most important consideration is the domain of definition of the functions $f(x), g(x)$ and not the presence or absence of nonnegativity constraints. In the most general case, we may suppose $f(x), g(x)$ defined over a closed convex set $D$. Then, if we can find any point $x^{2}$ satisfying (2.3), with the condition $x^{2} \geqslant 0$ replaced by the condition that $x^{2}$ belong to $D$, the conclusion that $x^{\circ}$ maximizes $f(x)$ follows as in part (a) of the proof of Theorem 1. The statement of (KTL) must be altered to read,

(KTLD)

$$
\begin{gathered}
\left(f_{x}^{o}+\lambda^{o} g_{x}^{o}\right)\left(x^{1}-x^{o}\right) \leqslant 0 \text { for all } x^{1} \text { in } D, \\
\lambda^{o} g\left(x^{o}\right)=0, \\
\lambda^{o} \geqslant 0 .
\end{gathered}
$$

An analogue of (c) remains valid. In the context of a general domain of definition, $D$, the condition $f_{x}^{o} \neq 0$ should be altered to read

$$
f_{x}^{o}\left(x^{2}-x^{o}\right) \neq 0 \quad \text { for some } x^{2} \text { in } D .
$$

(If $D$ has the full dimensionality of the space, as in the case of the nonnegative orthant, this reduces to the condition, $f_{x}^{0} \neq 0$.) If the generalized form (2.3) does not hold, then for all $x^{2}$ in $D$,

$$
f_{x}^{o}\left(x^{2}-x^{o}\right) \geqslant 0 \text {. }
$$

In view of (4.1), we can write

$$
f_{x}^{o}\left(x^{2}-x^{o}\right)>0 \quad \text { for some } x^{2} \text { in } D .
$$

On the other hand, from (2.2) (with $x^{1} \geqslant 0$ replaced by the condition, $x^{1}$ in $D$ ) and (4.2),

$$
f_{x}^{o}\left(x^{1}-x^{0}\right)=0 \quad \text { for all } x^{1} \text { in the constraint set. }
$$

Then, for any $x^{1}$ in the constraint set, define

$$
\phi(u, v)=f\left[(1-u-v) x^{o}+u x^{1}+v x^{2}\right]-f\left(x^{o}\right) .
$$


Then it may easily be verified that $(2.12-14)$ still hold, and we seek to prove that $\phi(1,0) \leqslant 0$. The rest of the argument under (c) proceeds without change.

Finally, it is obvious that the argument for (d) of Theorem 1 requires no change. We can therefore state the following general theorem, which also incorporates the Remark to Theorem 1.

THEOREM 3: Let $f(x)$ be a differentiable quasi-concave function of the ndimensional vector $x$, and let $g(x)$ be an m-dimensional differentiable vector function, both defined for $x$ in a closed convex domain $D$. Let the set of vectors $x$ in $D$ for which $g(x) \geqslant 0$ be convex, let $x^{\circ}$ and $\lambda^{\circ}$ satisfy (KTLD), and let one of the following conditions be satisfied:

(a) $f_{x}^{o}\left(x^{2}-x^{0}\right)<0$ for some $x^{2}$ in $D$;

(b) $f_{x}^{o}\left(x^{2}-x^{o}\right) \neq 0$ for some $x^{2}$ in $D$ and $f(x)$ is twice differentiable in some neighborhood of $x^{0}$;

(c) $f(x)$ is concave.

Then $x^{\circ}$ maximizes $f(x)$ subject to the constraints, $g(x) \geqslant 0, x$ in $D$.

The analogue of Theorem 2 also holds. If $g\left(x^{*}\right)>0$ for some $x^{*}$ in $D$ and for each $j, g^{j}(x)$ is concave or quasi-concave and $g_{x}^{j o} \neq 0$ for all $x^{o}$ in the constraint set, (KTLD) are necessary for a constrained maximum.

(2) Equality Constraints.

The constraint $g(x)=0$ can be expressed by the two inequality constraints $g(x) \geqslant 0$ and $-g(x) \geqslant 0$. Thus, if $g(x)$ and $-g(x)$ are both quasi-concave, as they will be, for example, if $g(x)$ is linear, Theorem 1 can be applied.

In this case, the last two lines of (KTL) become simply $g\left(x^{o}\right)=0$.

There is no analogue of Theorem 2 here. However, we have already pointed out that if $g(x)$ is linear, (KTL) is necessary for a maximum.

(3) Unconstrained Maxima.

First, suppose that all variables must be nonnegative, but that there are no other constraints. Since all variables are relevant, conditions (a), (b), and (c) of Section 2 become $f_{x}^{o} \neq 0$ as in (1) above. (KTL) becomes $f_{x}^{o} \leqslant 0$, $x^{\circ} f_{x}^{o}=0$. These statements together imply that $x^{o}$ maximizes the quasiconcave function $f(x)$ for $x \geqslant 0$ if either (a) $f_{x}^{o} \leqslant 0, f_{x}^{o} \neq 0$, and $x^{o} f_{x}^{o}=0$, or (b) $f_{x}^{o}=0$ and $f(x)$ is concave. The first condition requires that the usual first-order conditions for a maximum be satisfied with at least one corner variable. In effect, the existence of the corner variable rules out such possibilities as that the apparent maximum was produced by a cubic transformation.

The Constraint Qualification is automatically satisfied in this case. Hence, for nonnegative variables, $f_{x}^{o} \leqslant 0, x^{o} f_{x}^{o}=0$ is necessary for an unconstrained maximum for any differentiable $f(x)$. 
If the variables are unrestricted, (KTL) becomes $f_{x}^{o}=0$. As the examples at the end of Section 1 show, no conclusion can be drawn in general from (KTL) unless $f(x)$ is concave in which case the condition is clearly sufficient for a maximum.

If the variables are restricted to a general convex domain $D$, then (KTLD) becomes

$$
f_{x}^{0}\left(x^{1}-x^{0}\right) \leqslant 0 \quad \text { for all } x^{1} \text { in } D .
$$

This condition will be sufficient for a maximum if one of (a), (b), or (c) of Theorem 3 holds.

\section{ECONOMIC APPLICATIONS}

(1) Consumer Demand.

The fundamental property of the utility function in the theory of consumer demand is that the indifference curves define convex sets or a diminishing marginal rate of substitution. Thus, the minimal property of all utility functions is quasi-concavity. The propositions of consumer demand theory such as the basic Weak Axiom of Revealed Preference follow directly from quasiconcavity without appeal to bordered determinants of partial derivatives, monotonic transformations and the like.

Let the utility function $u(x)$ be quasi-concave and assume non-satiation, that is $u_{x_{i}}^{o}>0$ for some $x_{i}$. Then the usual first order conditions are necessary and sufficient for a constrained maximum. Let $x^{o}$ satisfy the conditions

$$
\begin{array}{cl}
u_{x_{i}}^{o}-\lambda^{o} p_{x_{i}} \leqslant 0 & (i=1, \ldots, n), \\
x_{i}^{o}\left(u_{x_{i}}^{o}-\lambda^{o} p_{x_{i}}\right)=0 & (i=1, \ldots, n), \\
\lambda^{o}\left(B-\Sigma x_{i}^{o} p_{x_{i}}\right)=0 & (i=1, \ldots, n),
\end{array}
$$

where $p_{x_{i}}>0$ is the price of a unit of $x_{i}$ and $B$ is the consumer's budget. Then $x^{\circ}$ maximizes $u(x)$ subject to the constraints $B-\Sigma x_{i} p_{x_{i}} \geqslant 0$ and $x \geqslant 0$. Moreover, if $\lambda^{\circ}>0$, and the assumption of non-satiation assures that it will be, $x^{0}$ minimizes the cost of attaining $u\left(x^{o}\right)$, for it maximizes $-\Sigma x_{i} p_{x_{i}}$ subject to the constraints $u(x)-u\left(x^{0}\right) \geqslant 0$ and $x \geqslant 0 .{ }^{18}$

$18 u_{x_{i_{o}}}^{o}>0, p_{x_{i_{o}}}>0$, and $u_{x_{i_{o}}}^{o}-\lambda \circ p_{x_{i_{o}}} \leqslant 0$ imply $\lambda^{\circ}>0$. The first two lines of (KTL) for the second maximum problem are $-p_{x_{i}}+\mu^{o} u_{x_{i}}^{o} \leqslant 0$, and $x_{i}^{o}\left(-p_{x_{i}}+\mu^{o} u_{x_{i}}^{o}\right)$ $=0$, or (5.1) with $\mu^{o}=1 / \lambda^{0}$.

The sufficiency of (5.1) for consumers' demand theory is widely assumed; however, the only rigorous proof, under rather severe regularity conditions, is that of Wold [7, Theorem 6, p. 87]. 


\section{(2) Production.}

The theory of efficient production can now be extended to include production functions that are quasi-concave but not concave, that is to those cases in which there are increasing returns to scale but a diminishing marginal rate of substitution.

Suppose, for example, that an enterprise carries on production in a set of independent processes which transform purchased inputs into intermediate goods which are not traded on the market, and both into outputs. Let the scale or intensity of the $i$ th process be measured by the variable $x_{i}$. Let the $j$ th output or input into the $i$ th process be a monotonic function $g_{i j}\left(x_{i}\right)$ that is positive if the commodity in question is an output of the process, negative if it is an input. Number the final outputs $j=1, \ldots, m_{1}$, the purchased inputs, $j=m_{1}+1, \ldots, m_{2}$, the intermediate goods, $j=m_{2}+1, \ldots, m$, and let there be $n$ processes. Then the net output or input of the $j$ th commodity will be

$$
g_{j}(x)=\sum_{i=1}^{n} g_{i j}\left(x_{i}\right) .
$$

Now, consider the problem of deriving the minimum cost method of producing a fixed set of outputs at given input prices. Let the price of the $j$ th commodity be $p_{j}$. Then the problem is to maximize $\Sigma_{j=m_{1}+1}^{m_{2}} p_{j} g_{j}(x)$, subject to the output-level constraints $g_{j}(x)-g_{j}\left(x^{0}\right) \geqslant 0, j=1, \ldots, m_{1}$, and the constraints that the net outputs of the intermediate goods not be negative, or if we let $\mathscr{E}_{j}$ represent initial stocks, that the net consumption of intermediate goods not exceed the initial stocks, that is $g_{j}(x)+\mathscr{E}_{j} \geqslant 0, j=m_{2}+1, \ldots, m$.

Under what conditions will this problem satisfy the hypotheses of Theorem 1 ? Since any monotonic function of one variable is quasi-concave, the functions $g_{i j}\left(x_{i}\right)$ are quasi-concave. But here we encounter a difference between concave and quasi-concave functions which is important from the point of view of applications to economic theory. While nonnegative linear combinations of concave functions are also concave, nonnegative linear combinations of quasi-concave functions are not necessarily quasi-concave. As a consequence, the hypothesis of quasi-concavity cannot replace the stronger hypothesis of concavity in many parts of economic theory.

Consider one of the output constraints, $g_{j}(x)-g_{j}\left(x^{o}\right) \geqslant 0$, or

$$
\sum_{i=1}^{n} g_{i j}\left(x_{i}\right)-g_{j}\left(x^{0}\right) \geqslant 0 \text {. }
$$

For outputs, we have $g_{i j}^{\prime}\left(x_{i}\right) \geqslant 0$. If $g_{i j}^{\prime \prime}\left(x_{i}\right) \leqslant 0, g_{i j}\left(x_{i}\right)$ is concave, and therefore, so is $g_{j}\left(x_{i}\right)$. If $g_{i j}^{\prime \prime}\left(x_{i}\right)>0$ for one process, with $g_{i j}^{\prime \prime}\left(x_{i}\right) \leqslant 0$ for all the others, $g_{j}(x)$ can be, though is not necessarily, quasi-concave. If $g_{i j}\left(x_{i}\right)>0$ for two or more activities, $g_{j}(x)$ cannot be quasi-concave. For, if $g_{j}(x)$ is 
quasi-concave, the marginal rate of substitution between any pair of inputs must be diminishing, all other inputs held constant. That is, from (1.10), holding, for example, $x_{3}, \ldots, x_{n}$ constant,

$$
\left(g_{2 j}^{\prime}\right)^{2} g_{1 j}^{\prime \prime}+\left(g_{1 j}^{\prime}\right)^{2} g_{2 j}^{\prime \prime} \leqslant 0.19
$$

Thus, it is possible for either $g_{1 j}^{\prime \prime}$ or $g_{2 j}^{\prime \prime}$ to be positive without violating (1.10), but clearly both cannot be positive, and similarly for every other pair of processes. Therefore, $g_{i j}^{\prime \prime}\left(x_{i}\right)>0$ for at most one process if $g_{j}(x)$ is to be quasi-concave. The same is true for the constraints on the use of intermediate goods.

What about the maximand, $\sum_{j=m_{1}+1}^{m_{2}} p_{j} g_{j}(x)$ ? If the functions $g_{j}(x)$ are concave, their linear combination will be also. But if any are quasi-concave and not concave, the quasi-concavity of $\Sigma_{j=m_{1}+1}^{m_{2}} p_{j} g_{j}(x)$ cannot be guaranteed independently of the prices. Thus, the only way for Theorem 1 to be applicable for all sets of prices is for there to be diminishing or constant returns to scale in the use of the inputs. For any given set of prices we may, however, have a limited amount of increasing returns in the use of inputs measured in money terms.

On the other hand, to apply Theorem 1 to profit maximization, we maximize $\sum_{j=1}^{m_{2}} p_{j} g_{j}(x)$ subject to the constraints $g_{j}(x)+\mathscr{E}_{j} \geqslant 0, j=m_{2}+1$, $\ldots, m$. Now there can be a limited amount of increasing returns with regard to intermediate goods, but not, in general, with regard to outputs or to inputs purchased on the market (unless there is just one output and no purchased inputs). Again, for any given set of prices, a certain amount of increasing returns in outputs or purchased inputs measured in money can be tolerated.

Alternatively, let a firm's production function be

$$
Y=K^{\alpha} L^{\beta}
$$$$
(\alpha>0, \beta>0) \text {. }
$$

This function will be quasi-concave but not concave when $\alpha+\beta>1$. Then, Theorem 1 will apply to the problem of determining the efficient combination of inputs, given any specified output, but it will not be applicable to the profit maximization problem. That is, the problem of minimizing $r K+w L$, or of maximizing $-(r K+w L)$, where $r$ and $w$ are the cost of a unit of $K$ and $L$ respectively, subject to the constraints $Y-Y^{o} \geqslant 0, L \geqslant 0, K \geqslant 0$, satisfies the hypotheses of Theorem 1. But the problem of maximizing $\Pi(K, L)=$ $p K^{\alpha} L^{\beta}-r K-w L$, subject to $K \geqslant 0, L \geqslant 0$ does not satisfy the hypotheses of Theorem 1 because $\Pi(K, L)$ is not quasi-concave.

\section{(3) Welfare Economics.}

Suppose that society's over-all production possibility function is quasi-

19 The inequality (5.4) is a necessary condition for (5.3) to be quasi-concave but it is not sufficient. For the corresponding sufficient condition, see Section 6 , below. 
concave. The problem of determining an efficient allocation of resources (a Pareto optimum) can then be formulated as the problem of maximizing the utility of one household (a quasi-concave function) subject to the constraints (also quasi-concave) that total output is within society's production possibilities and that the utilities of all other households are at least equal to specified levels.

\section{PROPERTIES OF QUASI-CONCAVE FUNCTIONS}

In Section 1, we gave several alternative definitions of quasi-concavity. Although the equivalence of these definitions, or their relationships when they are not strictly equivalent, seems to be rather generally understood, we have been unable to find in the literature either a proof of the equivalence of quasi-concavity and diminishing marginal rates of substitution (or increasing marginal rates of transformation), or a statement of the relationship between quasi-concavity and the signs of the bordered determinants of partial derivatives of quasi-concave functions. ${ }^{20}$ Therefore, we provide both here.

Let $f(x)$ be a twice differentiable quasi-concave function, and let $x^{0}$ and $x^{1}$ be any two nonnegative vectors, not zero and not proportional to each other. Let

$$
g(u, v)=f\left(u x^{0}+v x^{1}\right), \quad u, v \geqslant 0 .
$$

Then $f(x)$ is quasi-concave if and only if $g(u, v)$ is quasi-concave for all $x^{0}$ and $x^{1}$. Clearly the quasi-concavity of $f(x)$ implies that of $g(u, v)$. On the other hand, if $g(u, v)$ is quasi-concave, then, in particular, for $0 \leqslant \theta \leqslant 1$, we have

$$
f\left[\theta x^{o}+(1-\theta) x^{1}\right]=g(\theta, 1-\theta) \geqslant \min [g(0,1), g(1,0)]=\min \left[f\left(x^{o}\right), f\left(x^{1}\right)\right] .
$$

If (6.2) holds for all $x^{0}$ and $x^{1}$, we have the quasi-concavity of $f(x)$ by definition.

Consider any locus of points on which $g(u, v)$ is constant. Along this locus, $d u / d v=-g_{v} / g_{u}$. If $f_{x}>0$ everywhere, as is normally the case in utility theory, $g_{u}>0$ and $g_{v}>0$, and $g_{v} / g_{u}$ is known as the marginal rate of substitution between the composite commodities $x^{0}$ and $x^{1}$. If $f(x)$ is quasiconcave, the marginal rate of substitution is diminishing. That is, $d\left(g_{v} / g_{u}\right) / d v$ $\leqslant 0$. If $f_{x}^{0}<0$ everywhere, as is normally assumed in production theory ( $-f_{x}$ being interpreted as marginal costs), $g_{u}<0$ and $g_{v}<0$, and $g_{v} / g_{u}$ is known as the marginal rate of transformation between the composite commodi-

20 Wold [7, Theorem 5, pp. 85-86] states the relation between the signs of the bordered determinants and convexity of indifference surfaces to the origin, which is equivalent to quasi-concavity (see Theorem 4), under conditions more restrictive than those studied here. 
ties $x^{0}$ and $x^{1}$, and if $f(x)$ is quasi-concave, the marginal rate of transformation is increasing. That is, $d\left(g_{v} / g_{u}\right) / d v \geqslant 0$.

In order to prove these statements, observe that

$$
\frac{d}{d v}\left(\frac{g_{v}}{g_{u}}\right)=\frac{1}{g_{u}^{3}}\left[g_{u}^{2} g_{v v}-2 g_{u} g_{v} g_{u v}+g_{v}^{2} g_{u u}\right] .
$$

Thus, if $g_{u}>0$, we have a diminishing marginal rate of substitution, or if $g_{u}<0$, we have an increasing marginal rate of transformation, if the expression in brackets in (6.3) is less than or equal to zero. Therefore, to prove our propositions, we shall prove the following theorem.

THEOREM 4: The twice differentiable function $g(u, v)$ with $g_{u}>0$ and $g_{v}>0$ everywhere, or $g_{u}<0$ and $g_{v}<0$ everywhere, is quasi-concave if and only if $g_{u}^{2} g_{v v}-2 g_{u} g_{v} g_{u v}+g_{v}^{2} g_{u u} \leqslant 0$.

Proof. Since $g_{u}$ and $g_{v}$ are both positive or both negative, the implicit relation $g(u, v)=c$ defines $u$ as a function of $v$. Let the function be

$$
u=h(v) \text {. }
$$

Consider the case in which $g_{u}>0$ and $g_{v}>0$. By hypothesis, $d^{2} u / d v^{2} \geqslant 0$ so that $h(v)$ is a convex function.

Let $\left(u^{0}, v^{0}\right)$ and $\left(u^{1}, v^{1}\right)$ be any two points on the level curve $g(u, v)=c$. Then

$$
u^{o}=h\left(v^{o}\right), \quad u^{1}=h\left(v^{1}\right) .
$$

Let $\left(u^{2}, v^{2}\right)=(1-\theta)\left(u^{o}, v^{o}\right)+\theta\left(u^{1}, v^{1}\right)$, for $0 \leqslant \theta \leqslant 1$. Then, from (6.5), and the convexity of $h(v)$,

$$
h\left(v^{2}\right) \leqslant(1-\theta) h\left(v^{o}\right)+\theta h\left(v^{1}\right)=(1-\theta) u^{o}+\theta u^{1}=u^{2} .
$$

If $g_{u}>0$, it follows from the definition of $h(v)$ that

$$
c=g\left[h\left(v^{2}\right), v^{2}\right] \leqslant g\left(u^{2}, v^{2}\right),
$$

so that $g\left(u^{0}, v^{0}\right)=g\left(u^{1}, v^{1}\right)$ implies

$$
g\left[(1-\theta)\left(u^{o}, v^{o}\right)+\theta\left(u^{1}, v^{1}\right)\right] \geqslant g\left(u^{o}, v^{o}\right) \quad(0 \leqslant \theta \leqslant 1) .
$$

Quasi-concavity follows immediately. Suppose $g\left(u^{1}, v^{1}\right)>g\left(u^{0}, v^{o}\right)$. Let $\theta^{\prime}$ be the largest value of $\theta$ for which

$$
g\left[(1-\theta)\left(u^{o}, v^{o}\right)+\theta\left(u^{1}, v^{1}\right)\right]=g\left(u^{o}, v^{o}\right) .
$$

Now, let $\left(u^{2}, v^{2}\right)=\left(1-\theta^{\prime}\right)\left(u^{0}, v^{0}\right)+\theta^{\prime}\left(u^{1}, v^{1}\right)$. If $0 \leqslant \theta \leqslant \theta^{\prime}$, we can write $(1-\theta)\left(u^{0}, v^{o}\right)+\theta\left(u^{1}, v^{1}\right)=(1-t)\left(u^{0}, v^{o}\right)+t\left(u^{2}, v^{2}\right)$ where $t=\theta / \theta^{\prime}$. Since $g\left(u^{2}, v^{2}\right)$ $=g\left(u^{0}, v^{0}\right)$, we have shown that

$$
g\left[(1-\theta)\left(u^{0}, v^{o}\right)+\theta\left(u^{1}, v^{1}\right)\right]=g\left[(1-t)\left(u^{o}, v^{o}\right)+t\left(u^{2}, v^{2}\right)\right] \geqslant g\left(u^{o}, v^{o}\right)
$$


for $0 \leqslant \theta \leqslant \theta^{\prime}$. On the other hand, by continuity and the definition of $\theta^{\prime}$,

$$
g\left[(1-\theta)\left(u^{o}, v^{o}\right)+\theta\left(u^{1}, v^{1}\right)\right]>g\left(u^{o}, v^{o}\right)
$$

for $\theta^{\prime}<\theta \leqslant 1$. Thus $g$ satisfies (1.5) and is therefore quasi-concave. The theorem can be proved in a similar manner in the case in which $g_{u}<0$ and $g_{v}<0$ and there is an increasing marginal rate of transformation.

Finally, we shall prove that the quasi-concavity of $g(u, v)$ implies

$$
g_{u}^{2} g_{v v}-2 g_{u} g_{v} g_{u v}+g_{v}^{2} g_{u u} \leqslant 0 \text {. }
$$

Consider any pair of points $\left(u^{0}, v^{0}\right)$ and $\left(u^{1}, v^{1}\right)$ such that $g\left(u^{0}, v^{o}\right)=g\left(u^{1}, v^{1}\right)$. From (1.7) we have

$$
\begin{aligned}
& \left(u^{1}-u^{0}\right) g_{u}^{o}+\left(v^{1}-v^{0}\right) g_{v}^{o} \geqslant 0, \\
& \left(u^{0}-u^{1}\right) g_{u}^{1}+\left(v^{0}-v^{1}\right) g_{v}^{1} \geqslant 0,
\end{aligned}
$$

which, when added, imply

$$
\left(g_{u}^{o}-g_{u}^{1}\right)\left(u^{1}-u^{0}\right)+\left(g_{v}^{o}-g_{v}^{1}\right)\left(v^{1}-v^{0}\right) \geqslant 0 .
$$

Let $k=u^{1}-u^{0}$. In the limit, for $k$ small enough, $v^{1}-v^{0}=-k g_{u}^{0} / g_{v}^{0}$. Substituting these relationships into (6.13) and dividing through by $-k^{2}$, we obtain

$$
\begin{aligned}
\frac{g_{u}\left(u^{o}+k, v^{o}-k\left(g_{u}^{o} / g_{v}^{o}\right)\right)}{k}-g_{u}\left(u^{o}, v^{o}\right) \\
-\frac{g_{u}^{o}}{g_{v}^{o}} \frac{g_{v}\left(u^{o}+k, v^{o}-k\left(g_{u}^{o} / g_{v}^{o}\right)\right)-g_{v}\left(u^{o}, v^{o}\right)}{k} \leqslant 0 .
\end{aligned}
$$

Taking limits as $k$ approaches zero, and multiplying both sides by $g_{v}^{2}$, we obtain (6.11).

Now consider the bordered determinant $D_{r}$ defined by (1.11). The relationship between the property of quasi-concavity and the signs of $D_{r}$ is given by the following theorem.

THEOREM 5: A sufficient condition for $f(x)$ to be quasi-concave for $x \geqslant 0$ is that the sign of $D_{r}$ be the same sign of $(-1)^{r}$ for all $x$ and all $r=1, \ldots, n$. A necessary condition for $f(x)$ to be quasi-concave is that $(-1)^{r} D_{r} \geqslant 0$, for $r=1, \ldots, n$, for all $x$.

Proof. We shall begin by proving the sufficient condition. If $(-1) r D_{r}>0$ for all $r$ for any point $x^{0}$, then, by the usual second-order conditions for a constrained maximum, $x^{0}$ is a strict local maximum of $f(x)$ subject to the constraint $f_{x}^{0} x=f_{x}^{0} x^{0} .{ }^{21}$ Let $x^{1} \geqslant 0$ be any point $x^{1}$ for which

$$
f_{x}^{0} x^{1} \leqslant f_{x}^{0} x^{0}
$$

21 See, for example, [5, pp. 376-379]. 
We shall prove that $f\left(x^{1}\right) \leqslant f\left(x^{0}\right)$, that is, that $x^{0}$ is a global constrained maximum subject to $(6.15)$. Let

$$
x(\theta)=(1-\theta) x^{o}+\theta x^{1},
$$

and

$$
F(\theta)=f[x(\theta)] .
$$

Then let $\theta_{o}$ be the largest value of $\theta$ for which $F(\theta)$ takes its minimum in the interval $[0,1]$. We shall show that $\theta_{o}<1$ leads to a contradiction.

If $0<\theta_{o}<1$, then $F^{\prime}\left(\theta_{0}\right)=0$ because $F\left(\theta_{o}\right)$ is a minimum. If $\theta_{o}=0$, then $F^{\prime}(0) \geqslant 0$, so that $f_{x}^{o}\left(x^{1}-x^{o}\right) \geqslant 0$. But from $(6.15), F^{\prime}(0) \leqslant 0$, so that $F^{\prime}(0)=0$. Hence, in either case, $F^{\prime}\left(\theta_{0}\right)=0$, or

$$
f_{x}^{\theta^{o}}\left(x^{1}-x^{0}\right)=0
$$$$
\text { if } 0 \leqslant \theta_{0}<1 .
$$

Since $x\left(\theta_{o}+h\right)-x\left(\theta_{o}\right)=h\left(x^{1}-x^{o}\right)$, it follows from (6.18) that

$$
f_{x}^{\theta_{o}}\left[x\left(\theta_{o}+h\right)-x\left(\theta_{o}\right)\right]=0 \text {. }
$$

But, by assumption, (6.19) implies that $x\left(\theta_{0}\right)$ is a strict local maximum of $f(x)$ subject to $f_{x}^{\theta^{o}} x=f_{x^{o}}^{\theta^{o}} x\left(\theta_{o}\right)$, so that $f\left[x\left(\theta_{o}+h\right)\right]<f\left[x\left(\theta_{o}\right)\right]$, for $h$ positive and sufficiently small. This contradicts the definition of $\theta_{0}$ as the minimum of $F(\theta)$. It follows that we cannot have $\theta_{o}<1$, so that $\theta_{o}=1$ and in particular, $F(1) \leqslant F(0)$, or $f\left(x^{1}\right) \leqslant f\left(x^{o}\right)$.

We have thus shown that any point $x^{\circ}$ is a global constrained maximum of $f(x)$ subject to the constraints $x \geqslant 0$ and

$$
f_{x}^{o} x \leqslant f_{x}^{o} x^{0}
$$

Now, let $x^{0}$ and $x^{1}$ be any two points, and let $x^{2}$ be a convex combination (that is, an internal weighted average) of them. Since $f_{x}^{2} x^{2}$ is an internal average of $f_{x}^{2} x^{o}$ and $f_{x}^{2} x^{1}$, it must be at least as great as the lesser. That is, we must have either $f_{x}^{2} x^{0} \leqslant f_{x}^{2} x^{1}$ or $f_{x}^{2} x^{1} \leqslant f_{x}^{2} x^{2}$. Since $x^{2}$ maximizes $f(x)$ subject to $f_{x}^{2} x \leqslant f_{x}^{2} x^{2}$, we must then have either $f\left(x^{2}\right) \geqslant f\left(x^{o}\right)$ or $f\left(x^{2}\right) \geqslant f\left(x^{1}\right)$, and, in either case

$$
f\left(x^{2}\right) \geqslant \min \left[f\left(x^{0}\right), f\left(x^{1}\right)\right],
$$

so that $f(x)$ is quasi-concave.

To prove the necessity condition, first consider any $x^{0}>0$. If $f_{x}^{o}=0$, $D_{r}=0$ and the necessity condition is automatically satisfied. If $f_{x}^{0} \neq 0$, consider the maximization of $f(x)$ subject to the constraint (6.20). Since all variables are relevant and (KTL) is satisfied at $x^{0}$ with $\lambda^{\circ}=1$, it follows from Theorem 1 that $x^{\circ}$ is the constrained maximum. Therefore, $x^{o}$ certainly is a local constrained maximum of $f(x)$ subject to $f_{x}^{o} x=f_{x}^{o} x^{o}$, for which the 
conditions $(-1)^{r} D_{r} \geqslant 0$ are necessary. ${ }^{22}$ By continuity, this condition also holds when $x^{0}$ has one or more components that are equal to zero. ${ }^{23}$

In Section V, we discussed a necessary condition for the quasi-concavity of a function of the form

$$
g(x)=\sum_{i=1}^{n} g_{i}\left(x_{i}\right) .
$$

We now apply Theorem 5 to obtain a necessary and sufficient condition. In this case, $g_{x_{i}} x_{j}=0$ for $i \neq j$. Let $g_{x_{i}}=g_{i}^{\prime}$, and $g_{x_{i} x_{i}}=g_{i}^{\prime \prime}$, and let

$$
P_{r}=\prod_{i-1}^{r} g_{i}^{\prime \prime} \text {. }
$$

Then, by expansion of $D_{r}$, it is easy to see that

$$
D_{r}=-\left(g_{r}^{\prime}\right)^{2} P_{r-1}+g_{r}^{\prime \prime} D_{r-1} \text {. }
$$

If we assume, for simplicity, that $g_{i}^{\prime \prime} \neq 0$ for all $i$, then $D_{r} / P_{r}=-\left(g_{r}^{\prime}\right)^{2} / g_{r}^{\prime \prime}+$ $\left(D_{r-1} / P_{r-1}\right)$.

Since $D_{1} / P_{1}=-\left(g_{1}^{\prime}\right)^{2} / g_{1}^{\prime \prime}$, it easily follows by induction that,

$$
(-1)^{r} D_{r} /(-1)^{r} P_{r}=-\sum_{i=1}^{r}\left(g_{i}^{\prime}\right)^{2} / g^{\prime \prime} \text {. }
$$

If $g_{i}^{\prime \prime}<0$ for all $i$, then $(-1)^{r} P_{r}>0$ for all $r$ and the right-hand side of (6.25) is positive, from which it follows that $(-1)^{r} D_{r}>0$ for all $r$, and $g(x)$ is quasi-concave, indeed, concave.

Suppose $g_{i}^{\prime \prime}>0$ for two or more values of $i$. By renumbering, we may suppose that $g_{1}^{\prime \prime}>0, g_{2}^{\prime \prime}>0$. Then $P_{2}>0$. From (6.25), with $r=2$, we will have $D_{2}<0$, so that $g(x)$ is not quasi-concave.

In the remaining case, $g_{i}^{\prime \prime}>0$ for exactly one value of $i$; we may suppose,

$$
g_{i}^{\prime \prime}<0(i<n), g_{n}^{\prime \prime}>0 .
$$

Then,

$$
(-1)^{r} P_{r}>0(r<n),(-1)^{n} P_{n}<0 .
$$

The right-hand side of (6.25) is positive for $r<n$; with the aid of (6.27) we have that $(-1)^{r} D_{r}>0$ for $r<n$. To insure quasi-concavity, it is sufficient that $(-1)^{n} D_{n}>0$. In view of (6.27) and (6.25), this is equivalent to

$$
\sum_{i=1}^{n}\left(g_{i}^{\prime}\right)^{2} / g_{i}^{\prime \prime}>0 \text {. }
$$

22 See [5], ibid.

${ }^{23}$ Let $x^{1}>0$ and $x(t)=(1-t) x^{0}+t x^{1}$. Then $x(t)>0$ for $t>0$, whence $(-1)^{r} D_{r}(t)$ $\geqslant 0$ for $t>0$, where $D_{r}(t)$ is $D_{r}$ evaluated at the point $x(t)$. From this it follows that $(-1)^{r} D_{r}(0) \geqslant 0$. 
Since the first $n-1$ terms are negative, this means that the last term must outweigh them all, or that $g_{n}^{\prime \prime}$ must be sufficiently small relative to $\left(g_{n}^{\prime}\right)^{2}$. This places an upper limit on the permissible rate of increasing returns in the $n$th process. The stronger the rate of diminishing returns in the other processes, the greater is the permissible rate of increasing returns in the $n$th process.

Stanford University and the RAND Corporation

\section{REFERENCES}

[1] Arrow, Kenneth J., Leonid Hurwicz, and Hirofumi Uzawa: “Constraint Qualifications in Maximization Problems," Naval Research Logistics Quarterly, forthcoming.

[2] Courant, Richard: Differential and Integral Calculus, New York: Interscience Publishers, 1936.

[3] Fenchel, Werner: Convex Cones, Sets and Functions, Logistics Research Project, Princeton University, September, 1953 (mimeographed).

[4] Kunn, H. W., ANd A. W. Tucker: "Nonlinear Programming," in J. Neyman (ed.), Proceedings of the Second Berkeley Symposium on Mathematical Statistics and Probability, University of California Press, Berkeley, 1951, pp. 481-492.

[5] Samuelson, Paul A.: Foundations of Economic Analysis, Cambridge, Harvard University Press, 1948.

[6] Slater, Morton: "Lagrange Multipliers Revisited: A Contribution to Non-Linear Programming," Cowles Commission Discussion Paper, Math. 403, November, 1950.

[7] Wold, Herman: Demand Analysis, New York: Wiley, 1953. 